\title{
REDUCED ORDER MODEL OF A BLENDED WING BODY AIRCRAFT CONFIGURATION
}

\author{
F. Stroscher ${ }^{1}$, Z. Sika ${ }^{2}$, and Ö. Petersson ${ }^{1}$ \\ ${ }^{1}$ Institute of Lightweight Structures, Technical University Munich \\ 15 Boltzmannstr., Garching b. München 85747, Germany \\ ${ }^{2}$ Czech Technical University in Prague \\ Faculty of Mechanical Engineering \\ 4 Technicka, Praha 6, Czech Republik
}

This paper describes the full development process of a numerical simulation model for the ACFA2020 (Active Control for Flexible 2020 Aircraft) blended wing body (BWB) configuration. Its requirements are the prediction of aeroelastic and flight dynamic response in time domain, with relatively small model order. Further, the model had to be parameterized with regard to multiple fuel filling conditions, as well as flight conditions. High efforts have been conducted in high-order aerodynamic analysis, for subsonic and transonic regime, by several project partners. The integration of the unsteady aerodynamic databases was one of the key issues in aeroelastic modeling.

\section{NOMENCLATURE}

\section{Symbols}

$A, B, C, D$
$A_{0}, A_{1}, A_{2}, D, E, R$
AIC
$c$
$C_{p}$
$F$
FJKS
$G$
$J$

State-space matrices

Minimum-state approximation matrices

Aerodynamic influence coefficients

Reference chord length

Unsteady pressure coefficients

Flight dynamic forces

Differentiation matrix from $k$-set to $j$-set degrees of freedom (DOF)

Spline matrix between aerodynamic and structural DOF

Aircraft inertia tensor 


$\begin{array}{ll}k & \text { Reduced frequency } \\ K & \text { Stiffness matrix } \\ m & \text { Aircraft mass } \\ M & \text { Mass matrix; flight dynamic moments } \\ \text { Ma } & \text { Mach number } \\ p & \text { Roll rate } \\ q & \text { Pitch rate } \\ Q & \text { Generalized aerodynamic forces (GAF) } \\ r & \text { Yaw rate } \\ s & \text { Laplace variable } \\ \text { SKJ } & \text { Integration matrix from } j \text {-set to } k \text {-set DOF } \\ T_{\Phi} & \text { Mode shape transformation matrix } \\ u & x \text {-velocity in body axes } \\ v & y \text {-velocity in body axes } \\ V & \text { Velocities in body axes } \\ V_{\infty} & \text { Free-stream velocity } \\ w & z \text {-velocity in body axes, vertical gust velocity } \\ x_{l} & \text { Lag states } \\ X & X \text {-position } \\ Y & Y \text {-position } \\ Z & Z \text {-position } \\ \alpha & \text { Angle of attack } \\ \beta & \text { Sideslip angle } \\ \delta & \text { Control surface deflection } \\ \eta & \text { Modal deflection } \\ \Theta & \text { Pitch angle } \\ \Psi & \text { Yaw angle, mode shapes on panel control points } \\ \Phi & \text { Roll angle, mode shapes on structural DOF } \\ \Omega & \text { Rates in body axes } \\ & \end{array}$

\section{Subscripts/Superscripts}

$\begin{array}{ll}a & \text { Aerodynamic axes } \\ \mathrm{AE} & \text { Aeroelastic } \\ b & \text { Body axes } \\ c & \text { Control modes } \\ \mathrm{CFD} & \text { Computational fluid dynamics (CFD) } \\ D & \text { Drag force } \\ e & \text { Elastic modes } \\ \mathrm{FD} & \text { Flight dynamic } \\ g & \text { Gust modes } \\ G & \text { Gust forces }\end{array}$




$\begin{array}{ll}h & \text { Rigid body }+ \text { elastic modes } \\ l & \text { Roll moment } \\ L & \text { Lift force } \\ m & \text { Pitch moment } \\ n & \text { Yaw moment } \\ r & \text { Rigid body modes } \\ T & \text { Trim } \\ Y & \text { Side force }\end{array}$

\section{INTRODUCTION}

Today, active control and load alleviation is in use in military and more limited in civil transport aircraft. However, this technique still promises significant improvements for fuel efficiency, fatigue life, and ride comfort. Some future aircraft configurations, like the BWB, might even rely on active control to remain airworthy. An unacceptably high vibration level in the cabin
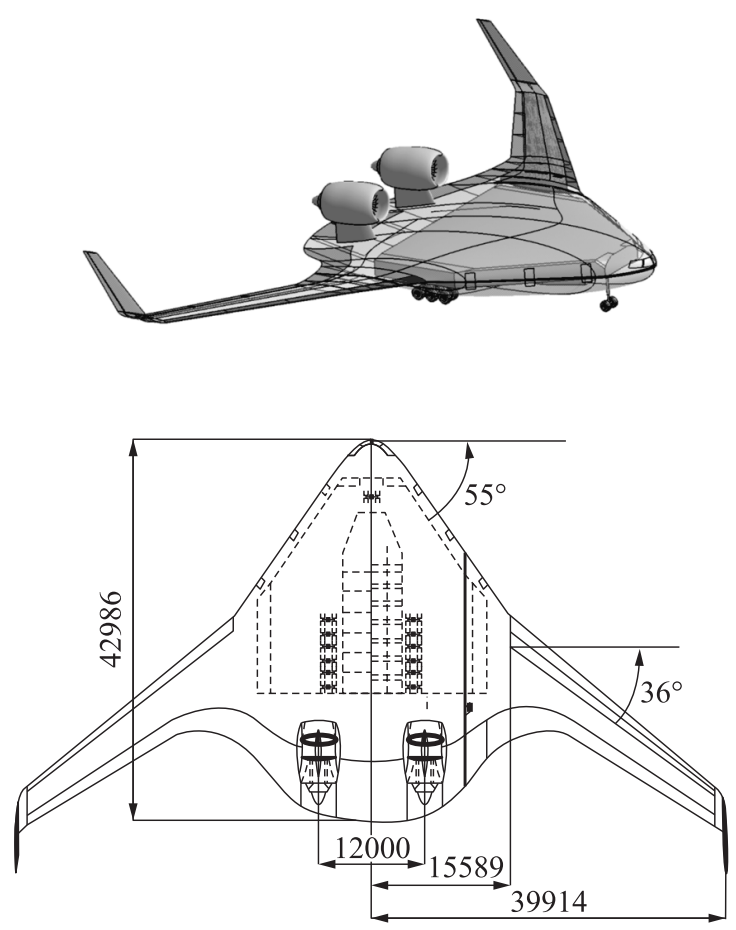

Figure 1 Exterior surface of BWB aircraft configuration. Dimensions are in millimeters 
of such aircraft might be reduced to the requirements on comfort of today aircraft, or even better. The ACFA2020 BWB aircraft configuration (Fig. 1) shall fully be exploited by active control design, in order to demonstrate best possible performance of load alleviation and ride comfort of such aircraft concepts.

Active control design optimization in aircraft configuration design demands for low-order simulation models, recovering the relevant aircraft dynamics for a manifold of mass distributions and flight conditions. Highly flexible aircraft show significant interaction of the rigid-body flight dynamics and aeroelastic dynamics, which poses challenging tasks in active control and load alleviation.

Thus, fully coupled flight dynamics and aeroelastic equations of motion (EOM) have to be employed. The full range of possible mass distributions, given by fuel tank filling, is captured by a parametric finite element model (FEM) of the aircraft primary structure. The coupled flight-dynamic and aeroelastic EOM are then set up, based on the eigendynamics of the FEM and a high-order CFD unsteady aerodynamic database. The parametric space of the model is augmented by multiple flight conditions, covering the whole flight envelope.

\section{STRUCTURAL MODEL PARAMETERIZATION}

Within the ACFA2020 project, a FEM of the BWB aircraft primary structure was developed and applied in aeroelastic modeling. The FEM level of detail is comparably low, but sufficient for the prediction of structural dynamic response

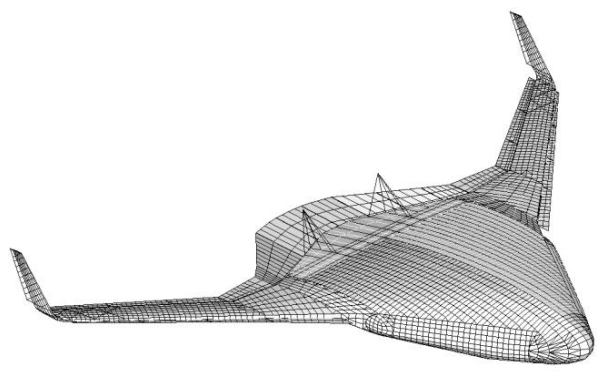

(a)

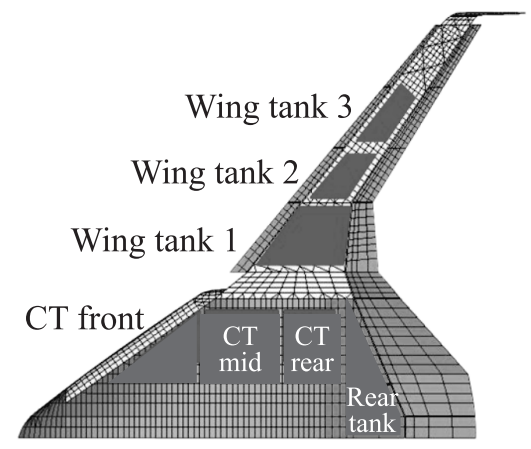

(b)

Figure 2 Finite element model of the BWB aircraft structure $(a)$ and fuel tank layout $(b)$ 
in the considered frequency range. A full span model was applied in aeroelastic modeling to directly take into account asymmetric turbulence excitation. The fuel tanks (Fig. 2) are represented by concentrated mass elements connected to the structure.

The possible loading conditions of the aircraft have been defined over the full range of fuel tank filling level, from empty to full. Further, the fuel distribution over tanks in wing and fuselage was considered, providing a useful margin of $x$-position of the aircraft center of gravity ( $\mathrm{CoG})$. Three configurations of fuel distributions are considered (CG1-3) for 11 steps of fuel filling (0: 0\%-10: 100\%). The range of possible CoG $x$-positions, measured from the aircraft nodes, depending on fuel filling, is shown in Fig. 3.

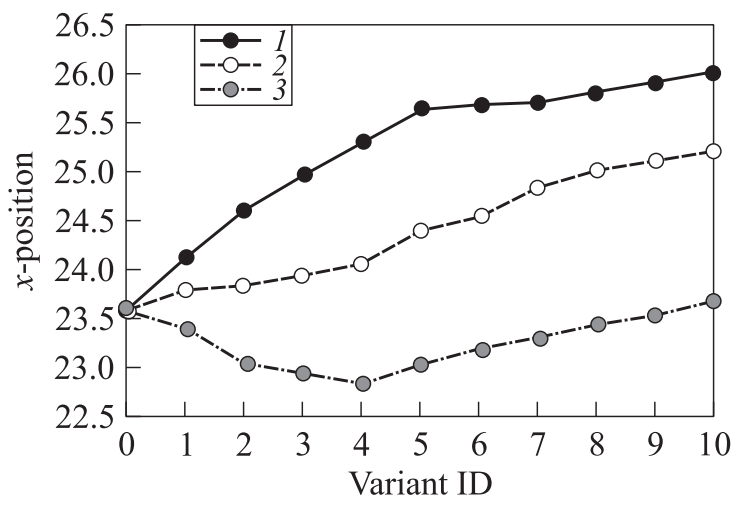

Figure 3 Center of gravity $x$-position of the considered mass variants: 1 - CG-1; $2-\mathrm{CG}-2$; and $3-\mathrm{CG}-3$

Modal analysis was performed for all mass configurations of the FEM, leading to individual modal bases applied in aeroelastic modeling (see subsection 4.1). The elastic modal deflections $\left[\Phi_{e}\right]$ are mass-normalized, leading to unitary modal mass matrices $\left[M_{\text {struct }}\right]$. To represent flight dynamics by the rigid body modes, its deflections are normalized to $1 \mathrm{~m}$ for translational modes, respectively, $1 \mathrm{rad}$.

\section{AERODYNAMIC ANALYSIS}

Steady aerodynamic analysis of the rigid aircraft as well as unsteady aerodynamic analysis of the flexible aircraft have been performed with simplified as well as high-order aerodynamic methods in subsonic and transonic regime. The databases have been combined and included in flight-dynamic and aeroelastic modeling. 


\subsection{Steady Aerodynamic Analysis of the Rigid Aircraft}

A steady aerodynamic database for the rigid BWB aircraft configuration was computed by project partners with different aerodynamic methods. Up to Mach number 0.6, a subsonic three-dimensional (3D) panel method was applied for computation of static polars, flap derivatives, as well as dynamic derivatives. The transonic regime was covered by steady aerodynamic analysis with CFD methods. Static polars and flap derivatives could be obtained by CFD analysis for a reduced set of angle of attack and sideslip angle conditions. However, dynamic derivatives have not been computed by CFD methods, thus had to be extrapolated from the subsonic aerodynamic database. The flap layout of the BWB aircraft, applied in aerodynamic analysis, is shown in Fig. 4.

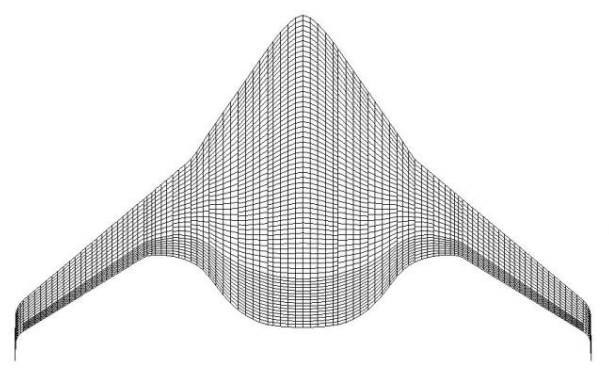

(a)

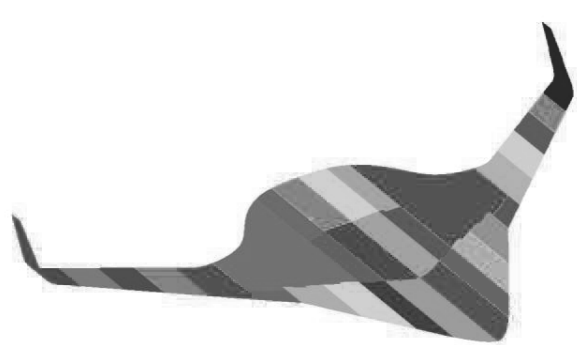

(b)

Figure 4 Panel mesh $(a)$ and subdivision into gust zones $(b)$

\subsection{Unsteady Aerodynamic Analysis of the Flexible Aircraft}

The unsteady aerodynamic analysis was performed with the subsonic panel method ZONA7, implemented in the aeroelastic toolkit ZAERO [1]. The lifting surfaces, the winglets, and the fuselage of the BWB aircraft have been modeled by wing panels, as shown in Fig. 4. Groups of panels in span and longitudinal directions build up gust zones, which account for individual gust downwash inputs in the simulation model.

As usual in aeroelastic analysis, the unsteady aerodynamic database is computed for modal coordinates, i. e., modal aerodynamic forces with regard to modal deflection. In order to account for the unsteady aerodynamics of control surfaces, additional control modes are introduced, which are defined by deflection of corresponding aerodynamic panels. Further, gust unsteady aerodynamic forces are included by a downwash distribution over the aircraft. 
The aeroelastic database is computed in frequency domain, assuming harmonic oscillation of mode shapes, control surface deflections, and gust downwash over a predefined frequency range. A nondimensional, so-called reduced frequency is applied here:

$$
\left\{C_{p}\right\}=\left[\operatorname{AIC}(i k, \mathrm{Ma}]^{\mathrm{T}}\{w\}\right.
$$

with $k=\omega c /\left(2 V_{\infty}\right)$.

A full matrix of aerodynamic influence coefficients for each panel, with regard to deflection of another panel is computed by the program.

Further detail on the unsteady aerodynamic foundation of ZAERO can be found in [1]. The complex AIC relate normal wash $w$ to unsteady pressure coefficients $C_{p}$ on aerodynamic panels, which are normalized by dynamic pressure. Note that the AIC are independent from structural properties and dynamic pressure.

Generalized aerodynamic forces, denoted by $Q$, are the unsteady aerodynamic forces on modal coordinates. By the use of an integration matrix SKJ, the pressure coefficients $C_{p}$ are converted to aerodynamic forces in the $6 \mathrm{DOF}$ directions of each panel at the panel control points [1]. For the transformation of the $6 \mathrm{DOF}$ displacements at the panels control points to normal wash, the differentiation matrix FJKS is employed. As the panel control points do not coincide with structural grid points, displacements and forces have to be transformed from structural to aerodynamic DOF by a spline matrix $G$. Now, the displacements are the generalized right-hand side by the modal matrices, defined on panel control points:

$$
\left[Q_{h}(i k)\right]=\left[\begin{array}{l}
Q_{r}(i k) \\
Q_{e}(i k)
\end{array}\right]=\left[\begin{array}{ll}
\Phi_{r} & \Phi_{e}
\end{array}\right]^{\mathrm{T}}[G]^{\mathrm{T}}[\operatorname{SKJ}]^{\mathrm{T}}[\operatorname{AIC}(i k)]^{\mathrm{T}}\left[\begin{array}{llll}
\Psi_{r} & \Psi_{e} & \Psi_{c} & \Psi_{g}
\end{array}\right]
$$

with

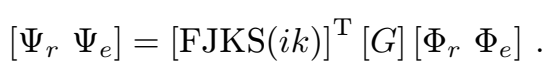

Here, $\Psi_{r}$ and $\Psi_{e}$ are computed from rigid-body modes $\Phi_{r}$ and structural modes $\Phi_{e}$, defined on structural DOF. The control modes $\Psi_{c}$ are directly defined on panel coordinates; $\Psi_{r}, \Psi_{e}$, and $\Psi_{c}$ are composed of real numbers, i. e., the deflection of the individual panel control points are in phase. Differently, the gust modes $\Psi_{g}$ have to be expressed by complex numbers, as there are variable phase angles for gust input on all aerodynamic panels. This approach takes into account that gusts downwash hits the individual parts of the lifting surface with correct time delays, as the gust is travelling through the aircraft.

Concluding, the aerodynamic forces are subdivided into columns for rigidbody, elastic, flap, and gust perturbations. Finally, the aerodynamic forces are 

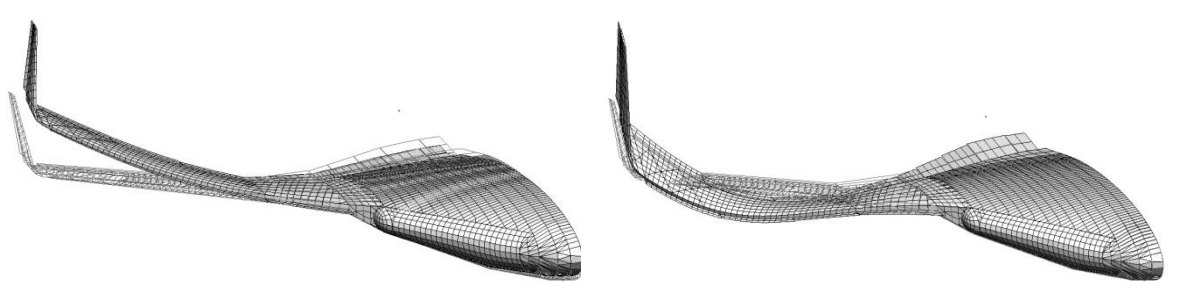

Figure 5 First two symmetric structural mode shapes

generalized left-hand side by the modal matrices for rigid-body modes and structural modes.

For the transonic regime, the generalized aerodynamic forces of some important structural modes have been corrected with results from an unsteady CFD simulation. Eight mode shapes, namely, the pitch and plunge mode, $\Phi_{r}^{\mathrm{CFD}}$, the first five symmetric and the first anti- symmetric mode shape, $\Phi_{e}^{\mathrm{CFD}}$, were chosen for CFD analysis. Due to limited computational resources, one intermediate mass configuration for the extraction of mode shapes for CFD analysis was chosen, meaning all fuel tanks were filled by $50 \%$. The resulting generalized aerodynamic forces were transformed afterwards to all other modal bases, used for the reduced order model (ROM). The first two symmetric mode shapes are shown in Fig. 5.

Further, the GAF from CFD analysis were computed for perturbations (righthand side generalization) of flaps $1,2,5,5^{\prime \prime}$, and 6 , represented by the flap modes $\Phi_{c}^{C F D}$. The projection (left hand side generalization) of the resulting unsteady pressures is now performed on the full set of rigid body and elastic mode shapes $\Phi_{h}^{\mathrm{CFD}}$ :

$$
\begin{aligned}
{\left[Q_{h}^{\mathrm{CFD}}(i k)\right]=\left[\Phi_{h}^{\mathrm{CFD}}\right]^{\mathrm{T}}[G]^{\mathrm{T}}[\mathrm{SKJ}]^{\mathrm{T}}[\mathrm{AIC}(i k)]^{\mathrm{T}} } & \\
\times & \times \operatorname{FJKS}(i k)]^{\mathrm{T}}[G]\left[\Phi_{r}^{\mathrm{CFD}} \Phi_{e}^{\mathrm{CFD}} \Phi_{c}^{\mathrm{CDF}}\right] .
\end{aligned}
$$

(Note that Eq. (1) represents an equivalent computation of GAF with the panel method, but does not reflect the process for CFD-computed GAF.)

Equation (1) yields a subset of columns of the full GAF with regard to the full modal basis. The same columns of the previously computed GAF are now replaced by the columns computed by $\mathrm{CFD}$ analysis. This results into a CFDcorrected GAF database with regard to the mode shapes of the intermediate mass configuration (CFD variant) of the aircraft structure. It should be noted that some correction factors had to be applied to the CFD-computed GAF columns, due to different modal deflection and the application of a half-span model in CFD analysis. 


\subsubsection{Transformation of computational fluid dynamics corrected generalized aerodynamic forces to structural mass variants}

The modified GAF for the high-speed aerodynamic regime are computed for the eight selected mode shapes, only. To recover these forces also for the modal bases of all 33 mass variants, a transformation rule for GAF has to be defined. Let $\left[Q_{h h}^{a}\right]$ be the GAF with regard to deflection of the mode shapes $\left[\Phi_{h}^{a}\right]$ and $\left[Q_{h c}^{a}\right]$ with regard to control surface deflection. Then, the GAF $\left[Q_{h h}^{b}\right]$ with regard to deflection of the mode shapes $\left[\Phi_{h}^{b}\right]$, and $\left[Q_{h c}^{b}\right]$ with regard to control surface deflection, are:

$$
\begin{aligned}
{\left[Q_{h h}^{b}(i k)\right] } & =\left[T_{\phi}^{b a}\right]^{\mathrm{T}}\left[Q_{h h}^{a}(i k)\right]\left[T_{\phi}^{b a}\right] ; \\
{\left[Q_{h c}^{b}(i k)\right] } & =\left[T_{\phi}^{b a}\right]^{\mathrm{T}}\left[Q_{h c}^{a}(i k)\right]
\end{aligned}
$$

with $\left[T_{\phi}^{b a}\right]$ as the least squares solution of the equation

$$
\left\lfloor\Phi_{h}^{a}\right\rfloor\left\lfloor T_{\phi}^{b a}\right\rfloor=\left\lfloor\Phi_{h}^{b}\right\rfloor .
$$

The above transformation yields a mathematically correct solution, only if a complete modal basis is used, meaning as many modes as DOF of the finite element model. Here, just 80 modes are inside the modal basis, but showed a sufficiently accurate solution of the transformation.

\subsubsection{Approximation of aerodynamic forces in the Laplace domain}

In order to derive equations of motion in the time-domain, the GAF are approximated in the Laplace-domain by the Minimum-State Method [2]. By replacing $i \omega$ with the Laplace variable $s$, the approximation equation in the Laplace domain becomes:

$$
\begin{aligned}
& {\left[Q_{h}(s)\right]=\left[\begin{array}{l}
Q_{r}(s) \\
Q_{e}(s)
\end{array}\right] } \\
= & {\left[\begin{array}{l}
A_{r 0} \\
A_{e 0}
\end{array}\right]+\frac{c}{2 V_{\infty}}\left[\begin{array}{l}
A_{r 1} \\
A_{e 1}
\end{array}\right] s+\left(\frac{c}{2 V_{\infty}}\right)^{2}\left[\begin{array}{l}
A_{r 2} \\
A_{e 2}
\end{array}\right] s^{2}+\left[\begin{array}{c}
D_{r} \\
D_{e}
\end{array}\right]\left([I] s-\frac{2 V_{\infty}}{c}[R]\right)^{-1}[E] s . }
\end{aligned}
$$

The rational function approximation is a well-known source of modeling errors, in particular, when approximating gust columns. An appropriate number of roots (size of $R$ ), as well as constraint setting for approximation (realand/or imaginary part fitting for one reduced frequency) is crucial for success.

The approximation matrices $A_{0}, A_{1}, A_{2}, D, E$, and $R$ are directly applied to the time-domain simulation model, to account for unsteady aerodynamic forces with regard to modal, control surface, and turbulence perturbations. 


\section{TIME-DOMAIN SIMULATION MODEL}

The simulation model was implemented in the well-known simulation environment Matlab Simulink, which was also preferably used for control design in the ACFA2020 project. The aerospace blockset was taken advantage of, for an easy implementation of some standard flight dynamics blocks, like the 6 DOF Newton-Euler EOM.

\subsection{Equations of Motion}

The time-domain simulation model represents the nonlinear 6 DOF NewtonEuler flight-dynamic equations of motion as well as aeroelastic equations of motion. Flight dynamics and structural (aeroelastic) dynamics are coupled by unsteady aerodynamic forces, whereas inertial coupling is not considered, assuming the aircraft dynamics on mean axes. For the sake of low model order, control surface dynamics are not directly computed in the simulation model, but represented by appropriate transfer functions from control commands to control deflection inputs. The flight dynamic DOF, $X, Y, Z, \Phi$, $\Theta$, and $\Psi$ are defined over body axes, as shown in Fig. 6, denoted by the subscript $B$.

The flight dynamics Newton-Euler EOM are the force and moment balance equations:

$$
\begin{aligned}
m\left\{\dot{V}_{b}+\Omega_{b} \times V_{b}-T_{b e} g_{e}\right\} & =F^{\mathrm{FD}}+F_{\mathrm{AE}}^{\mathrm{FD}}+F_{G}^{\mathrm{FD}} \\
J \dot{\Omega}_{b}+\Omega_{b} \times J \Omega_{b} & =M^{\mathrm{FD}}+M_{\mathrm{AE}}^{\mathrm{FD}}+M_{G}^{\mathrm{FD}}
\end{aligned}
$$

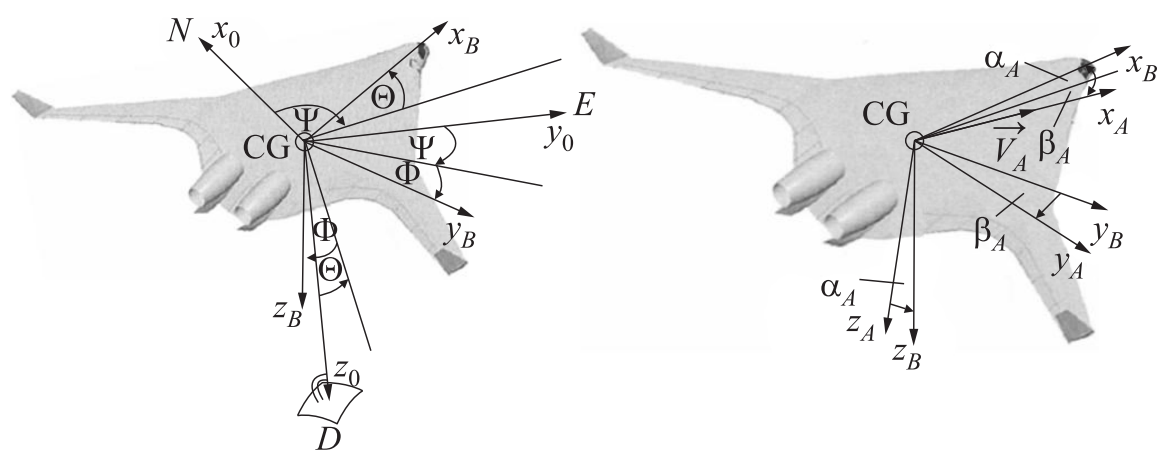

Figure 6 Definition of $N E D$-system $\left(x_{0}, y_{0}, z_{0}\right)$, body axes $\left(x_{B}, y_{B}, z_{B}\right)$, Euler angles $(\Phi, \Theta, \Psi)$, wind axes $\left(x_{A}, y_{A}, z_{A}\right)$, angle of attack $\alpha_{A}$, and sideslip angle $\beta_{A}$ 
with aerodynamic forces and moments due to flight dynamic motion,

$$
\begin{gathered}
F^{\mathrm{FD}} \\
=q_{\infty} S_{\mathrm{ref}} T_{b a}\left(\left\{\begin{array}{l}
C_{D} \\
C_{Y} \\
C_{L}
\end{array}\right\}+\left\{\begin{array}{l}
C_{D p} \\
C_{Y p} \\
C_{L p}
\end{array}\right\} p+\left\{\begin{array}{l}
C_{D q} \\
C_{Y q} \\
C_{L q}
\end{array}\right\} q+\left\{\begin{array}{l}
C_{D r} \\
C_{Y r} \\
C_{L r}
\end{array}\right\} r+\left\{\begin{array}{l}
C_{D \delta} \\
C_{Y \delta} \\
C_{L \delta}
\end{array}\right\} \delta\right) \\
M^{\mathrm{FD}}=q_{\infty} S_{\mathrm{ref}} \frac{c}{2} T_{b a}\left(\left\{\begin{array}{c}
C_{l} \\
C_{m} \\
C_{n}
\end{array}\right\}+\left\{\begin{array}{c}
C_{l p} \\
C_{m p} \\
C_{n p}
\end{array}\right\} p+\left\{\begin{array}{c}
C_{l q} \\
C_{m q} \\
C_{n q}
\end{array}\right\} q+\left\{\begin{array}{c}
C_{l r} \\
C_{m r} \\
C_{n r}
\end{array}\right\} r\right. \\
\left.+\left\{\begin{array}{c}
C_{l \delta} \\
C_{m \delta} \\
C_{n \delta}
\end{array}\right\} \delta\right)+(\mathrm{ARP}-\mathrm{COG}) \times F^{\mathrm{FD}},
\end{gathered}
$$

and aeroelastic coupling forces/moments and gust forces/moments,

$$
\begin{aligned}
& \left\{\begin{array}{l}
F_{\mathrm{AE}}^{\mathrm{FD}} \\
M_{\mathrm{AE}}^{\mathrm{FD}}
\end{array}\right\}=-q A_{r e 0} \eta-q_{\infty}\left(\frac{c}{2 V}\right) A_{r e 1} \dot{\eta}-q_{\infty}\left(\frac{c}{2 V}\right)^{2} A_{r e 2} \ddot{\eta}-q_{\infty} D_{r} x_{a} ; \\
& \left\{\begin{array}{l}
F_{\mathrm{AE}}^{\mathrm{FD}} \\
M_{\mathrm{AE}}^{\mathrm{FD}}
\end{array}\right\}=-\frac{1}{V} q_{\infty} A_{r g 0} w_{g}-q_{\infty}\left(\frac{c}{2 V^{2}}\right) A_{r g 1} \dot{w}_{g} .
\end{aligned}
$$

The aeroelastic EOM are comprised by the modal inertial and elastic forces on the left-hand side, as well as aerodynamic forces due to flight-dynamic, elastic, control surface, and turbulence perturbation, on the right-hand side of the following equation:

$$
\begin{aligned}
& {\left[M_{\text {struct }}\right]\left\{\ddot{\eta}_{e}\right\}+\left[K_{\text {struct }}\right]\left\{\eta_{e}\right\}=\left[A_{e 0}\right]\left\{\begin{array}{c}
0 \\
\left\{\eta_{e}\right\} \\
\left\{\delta_{c}\right\} \\
\frac{1}{V_{\infty}}\left\{w_{g}\right\}
\end{array}\right\}} \\
& +\left(\frac{c}{2 V_{\infty}}\right)\left[A_{e 1}\right]\left\{\begin{array}{c}
\left\{\dot{\eta}_{r}\right\} \\
\left\{\dot{\eta}_{e}\right\} \\
\left\{\dot{\delta}_{c}\right\} \\
1 \\
V_{\infty}\left\{\dot{w}_{g}\right\}
\end{array}\right\}+\left(\frac{c}{2 V_{\infty}}\right)^{2}\left[A_{e 2}\right]\left\{\begin{array}{c}
\left\{\ddot{\eta}_{r}\right\} \\
\left\{\ddot{\eta}_{e}\right\} \\
0 \\
0
\end{array}\right\}+\left[D_{e}\right]\left\{x_{l}\right\} .
\end{aligned}
$$

The rigid-body modes $\eta_{r}$ account for aerodynamic forces due to flightdynamic motion $[3,4]$. For the coupling of flight dynamic motion to rigid body modes, $\eta_{r}$ has to be expressed by the flight dynamic DOF: 


$$
\eta_{r}=\left(\begin{array}{c}
-X \\
Y \\
-Z \\
-\Phi \\
\Theta \\
-\Psi
\end{array}\right) ; \quad \dot{\eta}_{r}=\left(\begin{array}{c}
-u \\
v \\
-w \\
-p \\
q \\
-r
\end{array}\right) ; \quad \ddot{\eta}_{r}=\left(\begin{array}{c}
-\dot{u} \\
\dot{v} \\
-\dot{w} \\
-\dot{p} \\
\dot{q} \\
-\dot{r}
\end{array}\right)
$$

The aeroelastic EOM are augmented by a lag equation, which accounts for time delays of unsteady aerodynamic forces:

$$
\left\{\dot{x}_{l}\right\}=\left(\frac{2 V_{\infty}}{c}\right)[R]\left\{x_{l}\right\}+[E]\left\{\begin{array}{c}
\left\{\dot{\eta}_{r}\right\} \\
\left\{\dot{\eta}_{e}\right\} \\
\left\{\dot{\delta}_{c}\right\} \\
\frac{1}{V_{\infty}}\left\{\dot{w}_{g}\right\}
\end{array}\right\} .
$$

The first-order differential equation is solved for the lag states, which are coupled to the aeroelastic EOM, Eq. (2).

Unsteady aerodynamic forces are computed for rigid-body and elastic modes (see section 3), which are defined on a body fixed coordinate system (Fig. 7). Thus, the aerodynamic response of the rotational rigid-body modes about $y$ and $z$-axis is equal to a corresponding change in angle of attack $\alpha$ or sideslip angle $\beta$. Therefore, aircraft attitude $(\Phi, \Theta, \Psi)$, which does not change the aircraft heading with regard to the airflow, may not be represented by the rotational

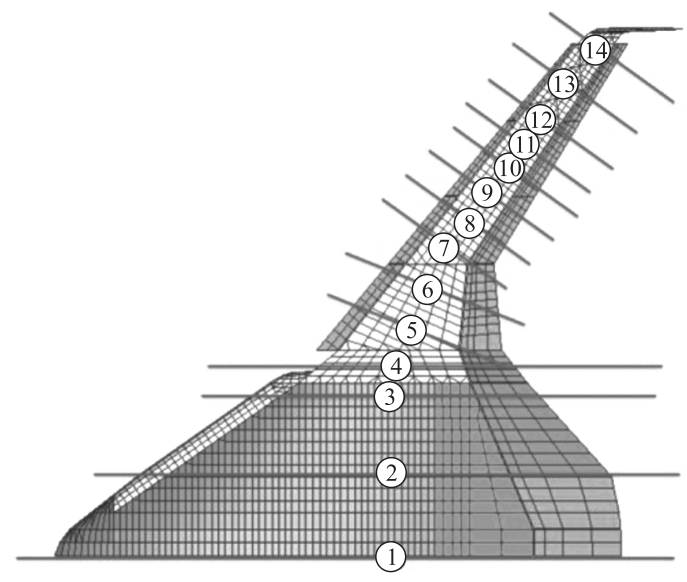

(a)

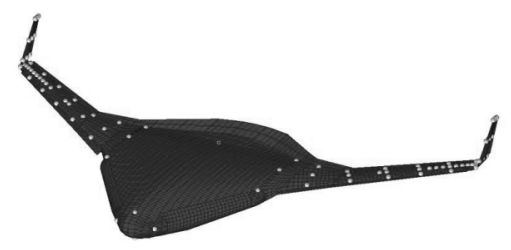

(b)

Figure 7 Positions over wingspan for cut forces outputs $(a)$ and acceleration sensor positions over airframe $(b)$ 
rigid-body modes. Instead, perturbations of $\alpha$ and $\beta$ are taken into account by velocity proportional aerodynamic forces of the rigid-body translations in $y$ - and $z$-axis directions. Note that the contribution of $A_{e 0}$ to the aerodynamic coupling from flight dynamics to structural dynamics is omitted in Eq. (2) for the above described reason [3].

\subsection{Sensor System}

Besides flight dynamic measurement outputs, like alpha/beta probes and CoG accelerations, structural sensors are integrated into the model, to be applied for load alleviation in active control design. These are several acceleration sensors (with regard to body axes) distributed over wingspan and wingchord, as well as cutforce and moment sensors at 14 positions over wingspan for left and right part of the aircraft (see Fig. 7).

\subsection{Trimming and Linearization}

In ACFA2020, the requirement for control design was a linear state-space model of the BWB aircraft of reasonably low order. Thus, the nonlinear simulation model was linearized at trimmed steady horizontal flight condition. The considered operating points are all possible combinations of fuel filling, CoG position, Mach number, and dynamic pressure. For each of them, a linear state-space model, covering flight-dynamic and aeroelastic response, as well as required inputs and outputs, was derived. The operating point state and input vectors for linearization are computed by a trim routine.

The trim routine is divided into two steps:

(i) flight dynamics longitudinal trim:

$$
\begin{aligned}
m g=C_{L 0}\left(\alpha_{T}, \beta=0, \mathrm{Ma}\right)+2 C_{L \delta_{1}}\left(\alpha_{T}, \beta=0, \mathrm{Ma}\right) \delta_{T} & \\
& +2 C_{L \delta_{2}}\left(\alpha_{T}, \beta=0, \mathrm{Ma}\right) \delta_{T} ; \\
m g\left(x_{\mathrm{ARP}}-\right. & \left.x_{\mathrm{CoG}}\right)=C_{m 0}\left(\alpha_{T}, \beta=0, \mathrm{Ma}\right)+2 C_{m \delta_{1}}(\alpha, \beta=0, \mathrm{Ma}) \delta_{T} \\
& +2 C_{m \delta_{2}}\left(\alpha_{T}, \beta=0, \mathrm{Ma}\right) \delta_{T}+2\left(z_{\mathrm{ARP}}-z_{\text {engine }}\right) F_{\text {thrust }} ;
\end{aligned}
$$

(ii) steady aeroelastic trim:

$$
\eta_{T}=-K_{e e}^{-1} q_{\infty}\left(\frac{c}{2 V}\right) A_{e r 1}\left\{\begin{array}{c}
V_{b, T} \\
\Omega_{b, T}
\end{array}\right\}
$$


where

$$
V_{b, T}=\left\{\begin{array}{c}
\cos \left(\alpha_{T}\right) V_{\infty} \\
0 \\
\sin \left(\alpha_{T}\right) V_{\infty}
\end{array}\right\} ; \quad \Omega_{b, T}=\left\{\begin{array}{l}
0 \\
0 \\
0
\end{array}\right\} .
$$

Lateral trim was not conducted, because no asymmetries of neither the aircraft nor its flight path had to be considered. To satisfy the longitudinal moment and lift equations, the elevators (flaps 1 and 2) and angle of attack were used as trim variables.

Equations (3)-(6) are solved iteratively until convergence of the trimmed angle of attack $\alpha_{T}$ and the trimmed elevator angle $\delta_{T}$. The resulting trim input and state vectors is applied as operating condition for linearization.

\subsection{Order Reduction}

There are several methods how to reduce the order of the time-domain simulation model suitable for the control algorithm design $[5,6]$. In spite of the different reduction concepts, there are always two basic possibilities, either the state truncation, denoted by subscript ST, or the singular perturbation approximation, denoted by subscript SPA. Let the original state space model be divided as follows (states corresponding to index 1 are the preserved ones, 2 are the reduced ones):

$$
\left[\begin{array}{ll}
A & B \\
C & D
\end{array}\right]=\left[\begin{array}{lll}
A_{11} & A_{12} & B_{1} \\
A_{21} & A_{22} & B_{2} \\
C_{1} & C_{2} & D
\end{array}\right]
$$

Then, the reduced order state space model obtained by state truncation of any type is:

$$
\left[\begin{array}{ll}
A & B \\
C & D
\end{array}\right]_{\mathrm{ST}}=\left[\begin{array}{cc}
A_{11} & B_{1} \\
C_{1} & D
\end{array}\right]
$$

The singular perturbation approximation variant of the reduced model preserves the $D C$-gains of an original system and generally can be written as

$$
\left[\begin{array}{ll}
A & B \\
C & D
\end{array}\right]_{\mathrm{SPA}}=\left[\begin{array}{cc}
A_{11}-A_{12} A_{22}^{-1} A_{21} & B_{1}-A_{12} A_{22}^{-1} B_{2} \\
C_{1}-C_{2} A_{22}^{-1} A_{21} & D-C_{2} A_{22}^{-1} B_{2}
\end{array}\right] .
$$

The preserving of the transfer functions for $D C$-gains and for the low frequencies is important in context of the low-order model approximation and, consequently, preserving of the rigid body and flight dynamics motion components. Therefore, the SPA [7] variant of the reduction has been always chosen.

The concept focused on four-order reduction is the balanced reduction [6] based on the given inputs and outputs with the chosen model dimension. This 

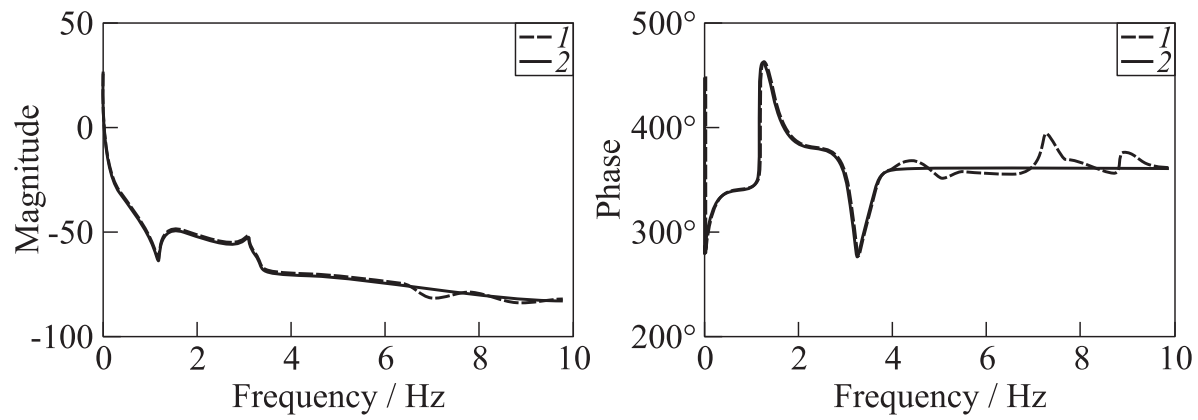

Figure 8 Comparison between original (1) and reduced (2) transfer functions

variant has been used as the reference one. The balanced reduction is very straightforward especially for the pure structural models with the proportional damping. The airplane model, however, also consists of the states with the high damping and even real poles. The evaluation of the final damping values of particular modes is relatively complex. The computational experiments show that the pure balanced reduction can discard some important states, like the first bending mode, some of the lag states, or even some flight mechanic states. Therefore, also other approaches have been applied.

The first variant of the control design ROM contains all flight mechanics states, all lag states, and states of the first (lowest) 19 elastic modes. The simplified variant of the control design ROM takes separately the symmetrical and antisymmetrical components including all flight mechanics states, all lag states, and states of the first (lowest) 4 elastic modes. The example of comparison between original ( 80 states) and reduced input-output (only 2 symmetrical modes) transfer functions is shown in Fig. 8.

\section{CONCLUDING REMARKS}

The results of aerodynamic and structural dynamic analysis of the BWB aircraft configuration have been applied to a flight-dynamic and aeroelastic modeling process. An easily applicable way for integrating results of unsteady CFD analysis into the full aerodynamic database was found. Aerodynamic coupling of flight dynamics and aeroelasticity, which is a key issue for flexible aircraft dynamics modeling, was accounted for by representing flight-dynamic motion by rigidbody modes. The time-domain equations of motion have been augmented by two-dimensional turbulence and control surface inputs as well as flight-dynamic and structural outputs. 
In a second step, the simulation model was trimmed and linearized at predefined operating points over the flight envelope. A model order reduction was applied to the linear models to provide a sufficiently accurate ROM for the control design process.

\section{REFERENCES}

1. ZONA Technology. 2008. ZAERO Theoretical Manual.

2. Karpel, M. 1981. Design for active and passive flutter suppression and gust alleviation. Technical Report CR-3482. NASA.

3. Looye, G. 2005. Integration of rigid and aeroelastic aircraft models using the residualised model method. Forum (International) on Aeroelastisity and Dynamics. München, Germany.

4. Kier, T. M., and G.H. Looye. 2009. Unifying manoeuvre and gust loads analysis models. Forum (International) on Aeroelastisity and Dynamics. Seattle, USA.

5. Obinata, G. O., and B.D. O. Anderson. 2001. Model reduction for control system design, communications and control engineering. Springer.

6. Gawronski, W. K. 2004. Advanced structural dynamics and active control of structures. N.Y.: Springer-Verlag, Inc.

7. Šika, Z, J. Zavřel, and M. Valášek. 2009. Residual modes for structure reduction and efficient coupling of substructures. Bull. Appl. Mech. 5(19):54-59. 